\title{
Pesquisas em Biotecnologia no Brasil: Uma correlação espacial entre índice-H e desenvolvimento social
}

\author{
Biotechnology research in Brazil: A spatial correlation between H-index and social development \\ Investiogación em Biotecnología em Brasil: Una correlación espacial entre el índice $\mathbf{H}$ y el
}

desarrollo social

Recebido: 08/01/2021 | Revisado: 11/01/2021 | Aceito: 12/01/2021 | Publicado: 15/01/2021

\author{
Igor Iuco Castro-Silva \\ ORCID: https://orcid.org/0000-0003-4815-6357 \\ Universidade Federal do Ceará, Brasil \\ E-mail: igor.iuco@sobral.ufc.br \\ Francisco Valdicélio Ferreira \\ ORCID: https://orcid.org/0000-0002-6347-2844 \\ Universidade Federal do Ceará, Brasil \\ E-mail: celionutri@gmail.com \\ Jacques Antonio Cavalcante Maciel \\ ORCID: https://orcid.org/0000-0002-2293-8433 \\ Universidade Federal do Ceará, Brasil \\ E-mail: jacques.maciel@ sobral.ufc.br
}

\begin{abstract}
Resumo
Indicadores bibliométricos são úteis para avaliar o impacto da produtividade acadêmica, aliados à compreensão geopopulacional. O objetivo deste estudo foi analisar a distribuição espacial de pesquisas em Biotecnologia no Brasil, verificando possível correlação entre índice-h e desenvolvimento social. A amostra foi composta por dados de 959 docentes e 59 programas strictu senso em Biotecnologia do país. Dados autorreferenciados de cada programa traçaram seus perfis descritivos. Foram construídos mapas de distribuição e de autocorrelação local (MoranMap) dos programas e do índice-h pela densidade populacional. Análise bivariada (LISA) considerou variáveis dependentes (taxa de programas e índice-h pela densidade populacional) e independente (IDHM). Como resultados, houve prevalência de doutorado acadêmico, com maior tempo de criação, carga horária, nota CAPES, número de docentes e índice-h médio, com maioria de programas na Região Sudeste, exceto mestrados acadêmicos, na Nordeste. A distribuição de programas pela densidade populacional foi maior em RIAUs de Palmas, Araraquara e Alfenas, enquanto de índice-h em RIAUs de Palmas, Belo Horizonte e Rio de Janeiro. Houve autocorrelação positiva de programas aglomerados em RIAUs de SP e Porto Alegre e entre programas e índice-h em RIAUs de Porto Alegre, Caxias do Sul e Ribeirão Preto. Análise bivariada IDHM-programas exibiu aglomerados alto-alto em RIAUs de SP e Porto Alegre e IDHM-índice-h aglomerados alto-alto em RIAUs de SP, MG e RJ. O mapeamento do índice-h e IDHM ajudou na compreensão da produtividade acadêmica da Biotecnologia no Brasil, contribuindo para discussão de modelos de avaliação em realidades desiguais no cenário nacional.
\end{abstract}

Palavras-chave: Indicadores de produção científica; Bases de dados bibliográficas; Sistemas de créditos e avaliação de pesquisadores; Biotecnologia.

\begin{abstract}
Bibliometric indicators are useful for assessing the impact of academic productivity, coupled with geopopulation understanding. The aim of this study was to analyze the spatial distribution of Biotechnology research in Brazil, verifying a possible correlation between the h-index and social development. The sample consisted of data from 959 professors and 59 stricto sensu biotechnology programs in the country. Self-referenced data for each program outlined their descriptive profiles. Local distribution and autocorrelation maps (MoranMap) of the programs and the $\mathrm{h}$-index by population density were built. Bivariate analysis (LISA) considered dependent variables (program rate and h-index for population density) and independent (MHDI). As a result, there was a prevalence of academic doctoral programs, with longer creation time, workload, CAPES score, number of teachers and average h-index, with most programs in the Southeast Region, except academic master's degrees, in the Northeast. The distribution of programs by population density was higher in RIAUs in Palmas, Araraquara and Alfenas, while the h-index in RIAUs in Palmas, Belo Horizonte and Rio de Janeiro. There was positive autocorrelation of programs clustered in RIAUs in SP and Porto Alegre and between programs and h-index in RIAUs in Porto Alegre, Caxias do Sul and Ribeirão Preto. Bivariate analysis MHDI-programs showed high-high clusters in RIAUs of SP and Porto Alegre and MHDI-h-index high-high clusters in RIAUs of SP, MG and RJ. The mapping of the h-index and MHDI helped to understand the academic
\end{abstract}


productivity of Biotechnology in Brazil, contributing to the discussion of evaluation models in unequal realities in the national scenario.

Keywords: Scientific publication indicators; Databases bibliographic; Researcher performance evaluation systems; Biotechnology.

\section{Resumen}

Los indicadores bibliométricos son útiles para evaluar el impacto de la productividad académica, junto con la comprensión geopopulacional. El objetivo de este estudio fue analizar la distribución espacial de la investigación biotecnológica en Brasil, verificando una posible correlación entre el índice h y el desarrollo social. La muestra estuvo conformada por datos de 959 profesores y 59 programas stricto sensu de biotecnología en el país. Los datos de autorreferencia para cada programa describieron sus perfiles descriptivos. Se construyeron mapas de distribución local y autocorrelación (MoranMap) de los programas y el índice h por densidad de población. El análisis bivariado (LISA) consideró variables dependientes (tasa de programa e índice h para densidad poblacional) e independientes (IDHM). Como resultado, prevalecieron los programas de doctorado académico, con mayor tiempo de creación, carga de trabajo, puntaje CAPES, número de docentes e índice h promedio, con la mayoría de los programas en la Región Sudeste, excepto maestrías académicas, en el Noreste. La distribución de los programas por densidad de población fue mayor en las RIAU de Palmas, Araraquara y Alfenas, mientras que el índice h en las RIAU de Palmas, Belo Horizonte y Río de Janeiro. Hubo una autocorrelación positiva de los programas agrupados en RIAU en SP y Porto Alegre y entre programas e índice h en RIAU en Porto Alegre, Caxias do Sul y Ribeirão Preto. Los programas de análisis bivariado MHDI mostraron conglomerados alto-alto en RIAU de SP y Porto Alegre y conglomerados alto-alto de índice MHDI-H en RIAU de SP, MG y RJ. El mapeo del índice h y el IDHM ayudó a comprender la productividad académica de la Biotecnología en Brasil, contribuyendo a la discusión de modelos de evaluación en realidades desiguales en el escenario nacional.

Palabras clave: Indicadores de producción científica; Bases de datos bibliográficas; Sistemas de créditos y evaluación de investigadores; Biotecnología.

\section{Introdução}

A divulgação de uma pesquisa é tão importante quanto a construção do saber, pois fomenta o debate dialético entre pesquisadores e oportuniza o compartilhamento de experiências aplicáveis para a comunidade, contribuindo para o bem-estar social (Simioni, Dallacorte \& Jacoski, 2016). A produtividade acadêmica é fenômeno antigo, onde o número de publicações atuava como fator determinante na progressão da carreira e reputação de um docente (De Paula e Boas, 2017). Este é um paradoxo entre ensino e pesquisa na atuação universitária, pois contribui para aumento de boas avaliações, de visibilidade e meritocracia em oportunidades institucionais e para adquirir fundos para pesquisas, apesar das pressões inerentes à sua jornada de produção (Alcadipani, 2017; Asnafi, Gunderson, McDonald \& Kallmes, 2017; Carpenter, Cone e Sarli, 2014; Doja et al., 2014). A boa conduta científica deve prevalecer sobre a cultura estadunidense do publish or perish, refutando a criação em série de artigos com dados repetitivos ou fraudulentos pelos pesquisadores, sem considerar o impacto de fato produzido na ciência (Alcadipani, 2017; Rigo, 2017).

Indicadores bibliométricos são medidas utilizadas para avaliação do impacto científico e destinados a informar o desempenho de pesquisadores numa dada área do conhecimento (Barreto, Aragão, Sousa, Santana e Barata, 2013). O campo da bibliometria oferece recursos multidimensionais aplicáveis a diferentes contextos, que inclui uso de bases eletrônicas, contagem total, download ou citações de artigos, bem como fatores de impacto ou percentis de citação de periódicos, que culminam na avaliação quantitativa da produção científica (Agarwal et al., 2016). A recuperação de publicações originais é facilitada por bancos de dados, como Web of Science, PubMed, PLoS, Scopus, ResearchGate ou Google Scholar, porém pode haver variações entre eles e exclusão de capítulos de livros, relatórios técnicos e artigos de conferências (Anker, Hadzibegovic, Lena \& Haverkamp, 2019; Bertoli-Barsotti \& Lando, 2017). Alguns dos indicadores bibliométricos mais aplicados mundialmente pela comunidade científica são: SCImago Journal Rank, JCR Impact Factor, índice-h e suas derivações, incluindo índice-h', índice-e, índice-h5, índice-hc, fator carbono_h, índice-p, índice-Ab, índice-Pr, índice-Bh, índice-v, dentre outros (Ciaccio et al., 2019; Carpenter et al., 2014; Bornmann, Marx, Gasparyan \& Kitas, 2012). 
O índice-h surgiu da busca por uma bibliometria focada no número de citações, a fim de superar a insuficiência da contagem total de artigos publicados e problemas com denominadores utilizados no cálculo de fator de impacto (Brandão e Mello, 2019; Hirsch, 2007; Hirsch 2005). A popularidade do índice-h é fruto da facilidade de obtenção, onde um pesquisador que tem 40 artigos publicados e recebe 25 citações terá o índice-h de 25 (Hirsch, 2005). Este índice fornece uma avaliação confiável e abrangente da significância de contribuições de um pesquisador, permitindo classificações entre indivíduos, grupos de trabalho, instituições, países, periódicos ou temas de pesquisa (Hirsch, 2019; Mester, 2016; Tabatabaei-Malazy, Ramezani, Atlasi, Larijani \& Abdollahi, 2016; Bornmann et al., 2012; Jacsó, 2009). Apesar do índice-h ter se tornado um dos indicadores mais utilizados para avaliação da produção científica e objeto de sérios debates relacionados às métricas na ciência (Cronin e Meho, 2006), seu criador, Jorge Hirsch, recomenda que um indicador bibliométrico deve ser usado sempre ao lado de outros indicadores e com bom senso (Marques, 2013).

A opção por índices internacionais de publicação poderia ser interpretado como provincianismo global ou colonialismo epistêmico pelo pensamento de Alcadipani (2017). Entretanto, diante da multiplicidade de indicadores, o índice-h pode ser generalizável, permitindo comparação com performances mundiais de produção científica (Hirsch, 2019; Hirsch, 2007; Hirsch, 2005). Similaridades entre resultados de índice-h pela busca em diferentes bases de dados (via Web of Science, Scopus ou Google Scholar) encontrados por Patel et al. (2013) com pesquisadores nas áreas de Fisiologia e Medicina e Barreto et al. (2013) com bolsistas de produtividade pelo CNPq aumentam o grau de confiança e reprodutibilidade deste indicador.

No início da década de 1980, o governo brasileiro fortaleceu investimentos na área de Biotecnologia, implementando o Programa Nacional de Biotecnologia junto a universidades públicas, mas também promovendo articulação da pesquisa com o setor privado (Dimova, Mitnik, Suarez-Buitron \& Siqueira, 2009). Conforme Torres-Freire, Golgher e Callil (2014), o Brasil presenciou um crescimento geral expressivo em recursos humanos na pós-graduação, aumentando exponencialmente entre 1987 e 2011 o número de mestres (onze vezes) e doutores (doze vezes), perfazendo 3.281 pesquisadores em Biotecnologia e Engenharia Biomédica ou 1,5\% do total do país. Porém, somente no fim da década de 2000, a CAPES deliberou abertura ou enquadramento de cursos stricto sensu em Biotecnologia, caracterizando-se como uma área transversal a várias ciências, atrativa de novos talentos, com potencial de crescimento e ainda recente no país (Brasil, 2017). Segundo León-de la O, Thorsteinsdóttir e Calderón-Salinas (2018), o Brasil manteve uma liderança editorial de publicações científicas em Biotecnologia sobre alguns países americanos, incluindo Argentina, Chile, Colômbia, Cuba e México, com crescimento de $380 \%$ no período entre 2001 e 2015.

A compreensão da regionalização da produtividade acadêmica biotecnológica permanece como uma lacuna do conhecimento, bem como possíveis fatores intrínsecos dos programas de pós-graduação ou extrínsecos de origem geopopulacional que possam interferir neste contexto. Por esse motivo, o objetivo deste estudo foi analisar a distribuição espacial de pesquisas em Biotecnologia no Brasil, hipotetizando uma possível correlação entre o índice-h e o desenvolvimento social.

\section{Metodologia}

Estudo de abordagem quantitativa, seguindo a tipologia ecológica ancorada em dados secundários (Pereira, Shitsuka, Parreira \& Shitsika, 2018). O cenário desse estudo abrangeu os programas de pós-graduação no Brasil cadastrados na Área Básica e de Avaliação em Biotecnologia na CAPES. A delimitação da amostra considerou como critérios de inclusão todos os programas stricto sensu na temática e seus respectivos docentes permanentes. Como critérios de exclusão, para descartar imprecisões geográficas ou humanas, não foram analisados: programas em rede com localização multicêntrica ou sem publicização em seus sites do corpo docente permanente, bem como docentes colaboradores, visitantes ou convidados, pois não integram o índice médio de produção qualificável para avaliação quadrienal dos programas. 
Os dados autorreferenciados de todos os programas foram coletados pelo acesso aberto à Plataforma Sucupira (Capes, 2020). Até 14 de junho de 2020, o número contabilizado de programas em Biotecnologia foi de 66, abrangendo 122 instituições, devido aos programas em rede, agregando um total de 959 docentes permanentes participantes. Os dados extraídos nesta plataforma foram: código do programa de pós-graduação, ano de criação, número de docentes permanentes, nível (mestrado acadêmico, mestrado profissional, doutorado acadêmico ou doutorado profissional), conceito CAPES e município sede. Através do site institucional de cada programa, fornecido pela plataforma, foi possível consultar individualmente o corpo docente atualizado publicitado. Foram excluídos da análise: dois programas que não apresentaram dados dos docentes em seus sites; cinco programas em rede (sem cidade sede, impossibilitando correlação com dados sociodemográficos municipais), perfazendo um total de 59 programas a serem analisados.

Para cada programa, o índice-h individual foi consultado através da base de dados Scopus (2020) até 14 de junho de 2020. A partir dos dados individuais, foi alcançada a média \pm desvio padrão do índice-h de docentes para cada programa.

Os dados de Índice de Desenvolvimento Humano Municipal (IDHM), um indicador de desenvolvimento social decenal, foram recuperados para cada município onde se localizam os programas de pós-graduação, através do Atlas br (2020). O IDHM pode ser classificado em muito baixo ( 0 a 0,499$)$, baixo (0,500 a 0,599), médio $(0,600$ a 0,699$)$, alto $(0,700$ a 0,799$)$ e muito alto (0,800 a 1), conforme o Programa das Nações Unidas para o Desenvolvimento (Pnud, 2003).

Os dados tabulados foram sumarizados em Excel $^{\mathrm{TM}}$ (Microsoft Office, EUA) e a amostra foi preliminarmente caracterizada por análise descritiva simples, com interpretação através de média e desvio padrão ou mediana e amplitude das variáveis: mestrado (acadêmico ou profissional) e doutorado (acadêmico ou profissional).

Foi realizada uma correlação espacial entre o índice-h e o IDHM de cada programa de pós-graduação em Biotecnologia. De acordo com o PNUD, foram encontrados IDHM nas faixas: baixo (0,500 a 0,599), médio $(0,600$ a 0,699$)$, alto $(0,700$ a 0,799$)$ e muito alto $(0,800$ a 1$)$. As informações foram georreferenciadas e foi realizada uma análise espacial a partir das malhas digitais das Regiões Intermediárias de Articulação Urbana (RIAU) pelo software GeoDa 0.9.9.10 ${ }^{\mathrm{TM}}$ (Spatial Analysis Laboratory, University of Illinois, EUA). Para a estimativa da autocorrelação espacial, utilizou-se o Índice de Moran Local, que varia entre -1 e +1 , e fornece sua significância estatística (valor de p). Após essa análise, foi avaliada a presença de aglomerados espaciais (clusters), a partir do Local Indicators of Spatial Association (LISA) e da construção de Mapa da autocorrelação local (MoranMap) relativos às variáveis dependentes e a média do IDHM da RIAU. Os mapas exibem aglomerados espaciais em quatro tipos: alto-alto (regiões formadas por municípios com altas frequências da variável, circundadas por regiões de altas frequências); baixo-baixo (regiões formadas por municípios com baixas frequências da variável, circundadas por regiões de baixas frequências), alto-baixo (regiões formadas por municípios com altas frequências da variável, circundadas por regiões de baixas frequências) e baixo-alto (regiões formadas por municípios com baixas frequências da variável, circundadas por regiões de alta frequência) (Maciel, Castro-Silva \& Farias, 2020).

Também foi realizada análise bivariada LISA para avaliação da correlação espacial entre a variável dependente (taxa de programas de pós-graduação em Biotecnologia e índice-h de profissionais para cada 100.000 habitantes) e a variável independente (IDHM), suavizados pela Estimativa Local de Bayes. Essa análise gerou o Índice de Moran Local e os mapas de correlação espacial (LISA). Para a correlação espacial bivariada, os clusters foram interpretados em um dos cinco tipos: não significativo (territórios que não entraram na formação de clusters, devido suas diferenças não terem sido significativas), altoalto (regiões formadas por municípios com altas frequências da variável dependente e altas frequências da variável independente), baixo-baixo (regiões formadas por municípios com baixas frequências da variável dependente e baixas frequências da variável independente), alto-baixo (regiões formadas por municípios com altas frequências da variável dependente e baixas frequências da variável independente) ou baixo-alto (regiões formadas por municípios com baixas 
frequências da variável dependente e altas frequências da variável independente). Os mapas temáticos foram elaborados com o software QGISTM 3.10 (Qgis, 2020).

Essa pesquisa foi realizada de acordo com os princípios bioéticos vigentes no Brasil para pesquisas envolvendo seres humanos e considerando o uso de dados secundários de acesso público sem possibilidade de identificação individual, especificados respectivamente pelas Resoluções do Conselho Nacional de Saúde nº. 466/2012 e nº. 510/2016.

\section{Resultados}

A Tabela 1 caracteriza a amostra obtida, destacando a grande prevalência de doutorado acadêmico (62,7\% do total) frente às demais modalidades de programas de pós-graduação em Biotecnologia no Brasil. Doutorados acadêmicos ( $\mathrm{n}=37)$, com 14,24 $\pm 10,54$ anos, 579,35 $\pm 235,14$ horas, nota CAPES mediana 4 (3-7), 18,4 $\pm 7,32$ docentes permanentes e índice-h médio de 6,17 a 27,38, foram mais concentrados na Região Sudeste, seguida pela Sul, Nordeste, Centro-Oeste e Norte. Doutorados profissionais $(\mathrm{n}=4)$, com 11,75 $\pm 5,56$ anos, 543,75 $\pm 140,91$ horas, nota CAPES mediana 5 (4-5), 14,25 $\pm 4,43$ docentes permanentes e índice-h médio de 5 a 8,57, foram mais concentrados na Região Sudeste do que na Sul e Nordeste. Mestrados

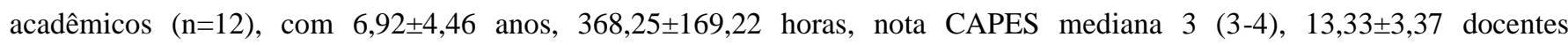
permanentes e índice h-médio de 6,17 a 27,46, foram mais concentrados na Região Nordeste, seguida pela Norte, Sudeste e

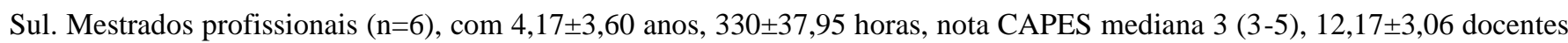
permanentes e índice-h médio de 3,2 a 11, tiveram distribuição equitativa nas Regiões Sudeste, Sul e Nordeste.

Tabela 1 - Dados dos programas de pós-graduação em Biotecnologia ativos no Brasil (n=59).

\begin{tabular}{|c|c|c|c|c|c|c|c|c|}
\hline Código CAPES (Instituição) & Nível & Criação & $\mathbf{C H}$ & CAPES & Dp & Índice $h^{*}$ & Cidade & IDHM \\
\hline 01. 12008010003P1 (UEA) & $\mathrm{ME}$ & 2003 & 375 & 3 & 11 & $8,72 \pm 2,95$ & Boca do Acre-AM & 0,588 \\
\hline 02. 22001018064P0 (UFC) & ME & 2008 & 684 & 4 & 16 & $8,81 \pm 2,96$ & Sobral-CE & 0,714 \\
\hline 03. 25001019081P0 (UFPE) & ME & 2010 & 360 & 4 & 12 & $10,75 \pm 3,27$ & Recife-PE & 0,772 \\
\hline 04. 15002012007P3 (UFRA) & ME & 2012 & 300 & 3 & 11 & $4,90 \pm 2,21$ & Belém-PA & 0,746 \\
\hline 05. 16003012011P0 (UFT) & ME & 2012 & 360 & 3 & 11 & $9,45 \pm 3,07$ & Gurupi-TO & 0,759 \\
\hline 06. $22021019001 P 2$ (UNINTA) & ME & 2014 & 720 & 3 & 10 & $7,10 \pm 2,66$ & Sobral-CE & 0,714 \\
\hline 07. 24001015074P1 (UFPB) & ME & 2014 & 330 & 3 & 6 & $8,16 \pm 2,85$ & João Pessoa-PB & 0,763 \\
\hline 08. 40001016083P6 (UFPR) & ME & 2015 & 270 & 3 & 13 & $27,46 \pm 5,24$ & Curitiba-PR & 0,823 \\
\hline 09. $27001016171 \mathrm{P5}$ (UFS) & ME & 2016 & 300 & 3 & 14 & $9,42 \pm 3,06$ & Sergipe-SE & 0,665 \\
\hline 10. $32006012171 P 1$ (UFU) & ME & 2017 & 120 & 3 & 15 & $6,66 \pm 2,58$ & Uberlândia-MG & 0,789 \\
\hline 11. 40006018176P6 (UTFPR) & ME & 2017 & 300 & 4 & 19 & $7,94 \pm 2,81$ & Curitiba-PR & 0,823 \\
\hline 12. $32011016044 P 0$ (UNIFAL) & ME & 2019 & 300 & A & 10 & $9,90 \pm 3,14$ & Alfenas-MG & 0,761 \\
\hline 13. 31001017013P2 (UFRJ) & DO & 1963 & 450 & 6 & 25 & $17,68 \pm 4,20$ & Rio de Janeiro-RJ & 0,761 \\
\hline 14. $33002010156 \mathrm{P0}$ (USP) & DO & 1991 & 570 & 5 & 43 & $18,76 \pm 4,33$ & São Paulo-SP & 0,783 \\
\hline 15. $42008018001 P 0$ (UCS) & DO & 1993 & 540 & 5 & 18 & $14,77 \pm 3,84$ & Caxias do Sul-RS & 0,782 \\
\hline 16. 33002088003P7 (USP) & DO & 1994 & 1080 & 5 & 14 & $15,64 \pm 3,95$ & Lorena-SP & 0,766 \\
\hline 17. $33004030077 P 0$ (UNESP) & DO & 1995 & 540 & 6 & 29 & $14,31 \pm 3,78$ & Araraquara-SP & 0,815 \\
\hline 18. $41001010035 P 8$ (UFSC) & DO & 1995 & 540 & 5 & 24 & $12,08 \pm 3,47$ & Florianópolis-SC & 0,847 \\
\hline 19. $40001016036 \mathrm{P8}$ (UFPA) & DO & 1997 & 540 & 7 & 13 & $27,38 \pm 5,23$ & Curitiba-PR & 0,823 \\
\hline 20. 33032017005P7 (UNAERP) & DO & 1999 & 540 & 4 & 11 & $13,27 \pm 3,64$ & Ribeirão Preto-SP & 0,800 \\
\hline 21. 33008019005P0 (UMC) & DO & 2000 & 540 & 4 & 16 & $10,18 \pm 3,19$ & Mogi das Cruzes-SP & 0,783 \\
\hline 22. 53003012005P4 (UCB) & DO & 2000 & 420 & 6 & 14 & $18,14 \pm 4,25$ & Taguatinga-DF & 0,634 \\
\hline 23. 40002012023P0 (UEL) & DO & 2001 & 570 & 5 & 13 & $10,46 \pm 3,23$ & Londrina-PR & 0,778 \\
\hline 24. 12001015013P9 (UFAM) & DO & 2001 & 600 & 4 & 34 & $10,32 \pm 3,21$ & Manaus-AM & 0,720 \\
\hline 25. $42003016020 P 2$ (UFPel) & DO & 2002 & 680 & 7 & 23 & $10,78 \pm 3,28$ & Pelotas-RS & 0,739 \\
\hline 26. 33001014020P4 (UFSCar) & DO & 2004 & 825 & 4 & 5 & $8,80 \pm 2,96$ & São Carlos-SP & 0,805 \\
\hline
\end{tabular}




\begin{tabular}{|c|c|c|c|c|c|c|c|c|}
\hline 27. 28002016006 P3 (UEFS) & DO & 2005 & 480 & 5 & 19 & $10,89 \pm 3,30$ & Feira de Santana-BA & 0,712 \\
\hline 28. 51002019005P2 (UCDB) & DO & 2006 & 450 & 4 & 14 & $17,57 \pm 4,91$ & Campo Grande-MS & 0,784 \\
\hline 29. 30001013029P3 (UFES) & DO & 2007 & 540 & 5 & 17 & $10,47 \pm 3,23$ & Vitória-ES & 0,845 \\
\hline 30. 32007019020P0 (UFOP) & DO & 2009 & 540 & 4 & 17 & $8,88 \pm 2,97$ & Ouro Preto-MG & 0,741 \\
\hline 31. 28001010080P6 (UFBA) & DO & 2010 & 340 & 4 & 10 & $10,00 \pm 3,16$ & Salvador-BA & 0,759 \\
\hline 32. 15001016068P6 (UFPA) & DO & 2011 & 390 & 5 & 16 & $12,00 \pm 3,46$ & Belém-PA & 0,746 \\
\hline 33. 27002012004P8 (UNIT) & DO & 2011 & 450 & 5 & 13 & $11,46 \pm 3,38$ & Aracajú-SE & 0,770 \\
\hline 34. 21001014025P2 (UFPI) & DO & 2011 & 720 & 4 & 17 & $10,64 \pm 3,26$ & Teresina-PI & 0,751 \\
\hline 35. $32018010011 P 9$ (UFSJ) & DO & 2011 & 300 & 4 & 22 & $8,36 \pm 2,89$ & São João Del Rei-MG & 0,758 \\
\hline 36. $33001014042 P 8$ (UFSCar) & DO & 2012 & 825 & 4 & 17 & $11,00 \pm 3,31$ & São Carlos-SP & 0,805 \\
\hline 37. 33107017008P0 (UNIAN-SP) & DO & 2012 & 450 & 3 & 10 & $8,10 \pm 2,83$ & São Paulo-SP & 0,783 \\
\hline 38. $33144010016 P 4$ (UFABC) & DO & 2012 & 528 & 4 & 22 & $14,40 \pm 3,79$ & Santo André-SP & 0,815 \\
\hline 39. 42014018003P9 (UNIVATES) & DO & 2012 & 600 & 4 & 12 & $12,66 \pm 3,55$ & Lajeado-RS & 0,778 \\
\hline 40. 22001018080P6 (UFC) & DO & 2013 & 768 & 4 & 21 & $18,04 \pm 4,24$ & Fortaleza-CE & 0,754 \\
\hline 41. 40004015042P7 (UEM) & DO & 2013 & 540 & 4 & 17 & $14,58 \pm 3,81$ & Maringá-PR & 0,808 \\
\hline 42. 31069010002P2 (INMETRO) & DO & 2013 & 570 & 4 & 13 & $12,53 \pm 3,53$ & Rio de Janeiro-RJ & 0,761 \\
\hline 43. $33009015085 P 0$ (UNIFESP) & DO & 2013 & 225 & 4 & 29 & $6,17 \pm 2,48$ & São Paulo-SP & 0,783 \\
\hline 44. $33004064087 P 8$ (UNESP) & DO & 2015 & 510 & 4 & 24 & $17,20 \pm 4,14$ & São Paulo-SP & 0,783 \\
\hline 45. 33082014005P6 (UNIARA) & DO & 2015 & 600 & 4 & 11 & $9,54 \pm 3,08$ & Araraquara-SP & 0,815 \\
\hline 46. $31033016018 P 2$ (UENF) & DO & 2016 & 595 & 4 & 16 & $16,37 \pm 4,04$ & Campos dos Goytacazes-RJ & 0,716 \\
\hline 47. 33009015170P7 (UNIFESP) & DO & 2016 & 600 & 4 & 23 & $11,69 \pm 3,41$ & São Paulo-SP & 0,783 \\
\hline 48. $33305005001 P 1$ (UFF) & DO & 2016 & 360 & 4 & 20 & $16,60 \pm 4,07$ & Niterói-RJ & 0,837 \\
\hline 49. 51001012177P1 (UFMS) & DO & 2019 & 1620 & A & 19 & $10,73 \pm 3,27$ & Campo Grande-MS & 0,784 \\
\hline 50. 42008018010P9 (UNISAGRADO) & MP & 2011 & 360 & 4 & 10 & $11,00 \pm 3,31$ & Caxias do Sul-RS & 0,782 \\
\hline 51. 42005019034P6 (PUCRS) & MP & 2013 & 360 & 5 & 9 & $10,33 \pm 3,21$ & Porto Alegre-RS & 0,805 \\
\hline 52. $32145012001 P 7$ (UNIFEMM) & MP & 2014 & 270 & 3 & 14 & $4,64 \pm 2,15$ & Sete Lagoas-MG & 0,760 \\
\hline 53. $21009015001 P 7$ (UniFacid) & MP & 2019 & 360 & 3 & 10 & $3,20 \pm 1,78$ & Teresina-PI & 0,751 \\
\hline 54. $28048016002 P 3$ (FAMAM) & MP & 2019 & 330 & A & 17 & $5,70 \pm 2,38$ & Gov. Mangabeira-BA & 0,643 \\
\hline 55. $32148011001 P 6$ (FUNED) & MP & 2019 & 300 & A & 13 & $9,07 \pm 3,11$ & Belo Horizonte-MG & 0,810 \\
\hline 56. $33004064079 P 5$ (UNESP) & DP & 2000 & 375 & 5 & 19 & $8,57 \pm 2,92$ & São Paulo-SP & 0,783 \\
\hline 57. 40022013004P9 (UP) & DP & 2010 & 540 & 5 & 10 & $6,20 \pm 2,48$ & Curitiba-PR & 0,823 \\
\hline 58. 32014015009P9 (UNIMONTES) & DP & 2011 & 540 & 4 & 17 & $5,00 \pm 2,23$ & Montes Claros-MG & 0,770 \\
\hline 59. $23004010004 P 0$ (UnP) & DP & 2012 & 720 & 4 & 11 & $5,72 \pm 2,39$ & Natal-RN & 0,763 \\
\hline
\end{tabular}

CH: carga horária. Dp: Docentes permanentes. *Média \pm desvio padrão. ME: Mestrado acadêmico, DO: Doutorado acadêmico. MP: Mestrado profissional. DP: Doutorado profissional. A: aberto, sem nota quadrienal.

Fonte: Autores, a partir de dados coletados em Capes (2020), Scopus (2020) e Atlas br (2020).

A distribuição dos programas de pós-graduação no Brasil com base na densidade populacional variou de 0 a 0,479 para cada 100.000 habitantes, com maior concentração nas RIAU de Palmas (TO), Araraquara (SP) e Alfenas (MG) (Figura 1a). O índice-h de docentes vinculados a estes programas também variou de 0 a 31 com maior concentração nas RIAU de Palmas (TO), Belo Horizonte (MG) e Rio de Janeiro (RJ) (Figura 1b). 
Figura 1 - Mapa da distribuição de programas de pós-graduação em Biotecnologia no Brasil (a) e índice-h dos programas pela densidade populacional (b).

a)

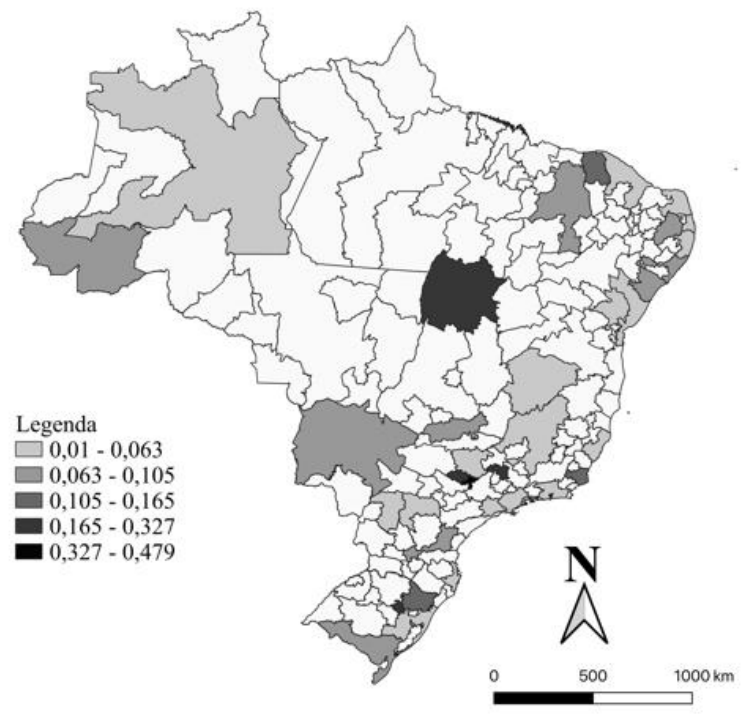

b)

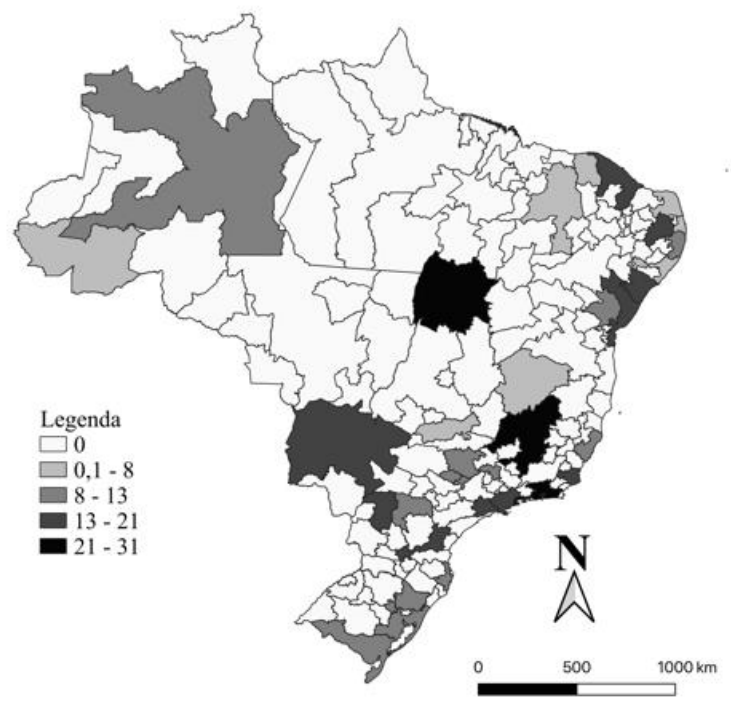

Fonte: Autores, a partir de dados coletados em Capes (2020), Scopus (2020) e Atlas br (2020).

As RIAU apresentaram autocorrelação espacial positiva da distribuição de programas de pós-graduação (Índice de Moran = 0,02 p<0,01), com formação de um aglomerado das RIAU de São Carlos, Ribeirão Preto e Araraquara (SP). A RIAU de Porto Alegre (RS) também apresentou padrão alto-alto (Figura 2a). Com base no índice-h, as RIAU apresentaram autocorrelação espacial positiva da distribuição de programas de pós-graduação (Índice de Moran = 0,07 p<0,01), com formação de um aglomerado das RIAU de Porto Alegre e Caxias do Sul (RS), assim como Ribeirão Preto (SP) (Figura 2b).

Figura 2 - Autocorrelação local (MoranMap) da distribuição de programas de pós-graduação em Biotecnologia no Brasil (a) e do índice-h dos programas pela densidade populacional (b).

a)

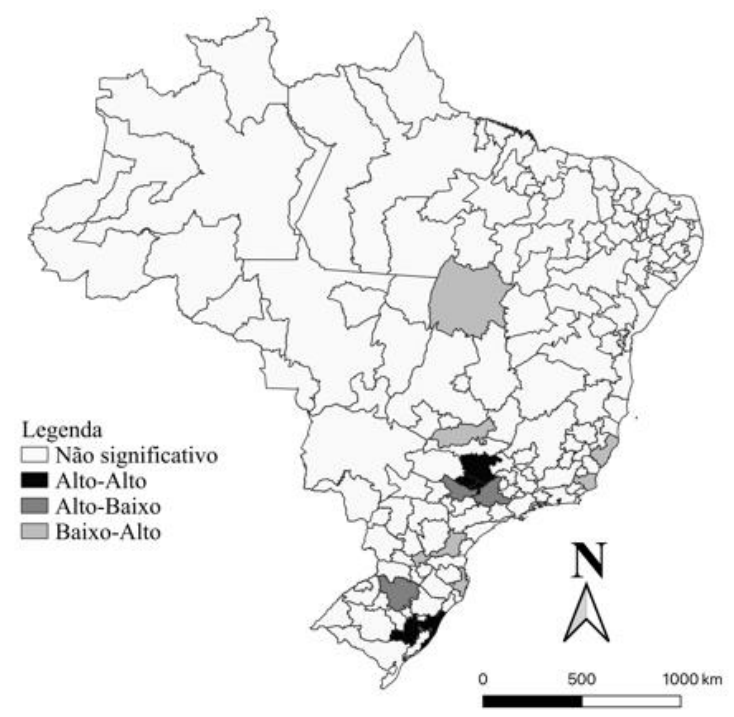

b)

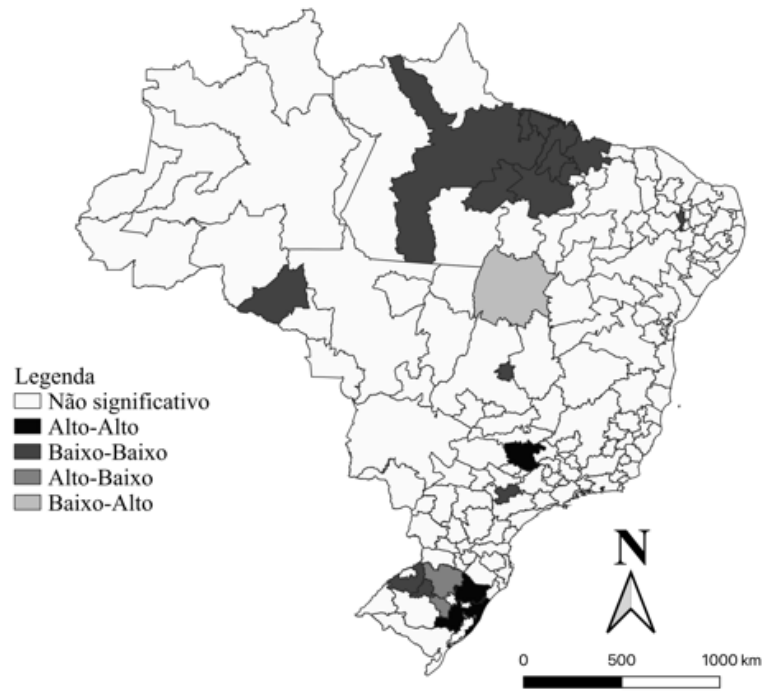

Fonte: Autores, a partir de dados coletados em Capes (2020), Scopus (2020) e Atlas br (2020).

No que se refere à análise de correlação espacial bivariada, pôde-se observar correlações positivas entre o número de programas de pós-graduação em Biotecnologia e IDHM (Índice de Moran = 0,118; p<0,01), ou seja, RIAU com um maior 
número de programas de pós-graduação com maiores valores de IDHM, com aglomerados alto-alto nas RIAU do estado de São Paulo (Araraquara, Ribeirão Preto, Bauru, Campinas e São Carlos) e de Porto Alegre (RS) (Figura 3a). Na análise do índice-h (Índice de Moran=0,153; p <0,01) as RIAU com padrão alto-alto foram dos estados de São Paulo (São José dos Campos e Santos), Minas Gerais (Passo Fundo, Passos, Divinópolis, Varginha, Juiz de Fora e Lavras) e Rio de Janeiro (Volta Redonda, Nova Friburgo e Macaé) (Figura 3b).

Figura 3 - Análise bivariada do Índice de Desenvolvimento Humano com a distribuição de programas de pós-graduação em Biotecnologia no Brasil (a) e o índice-h dos programas (b).

a)

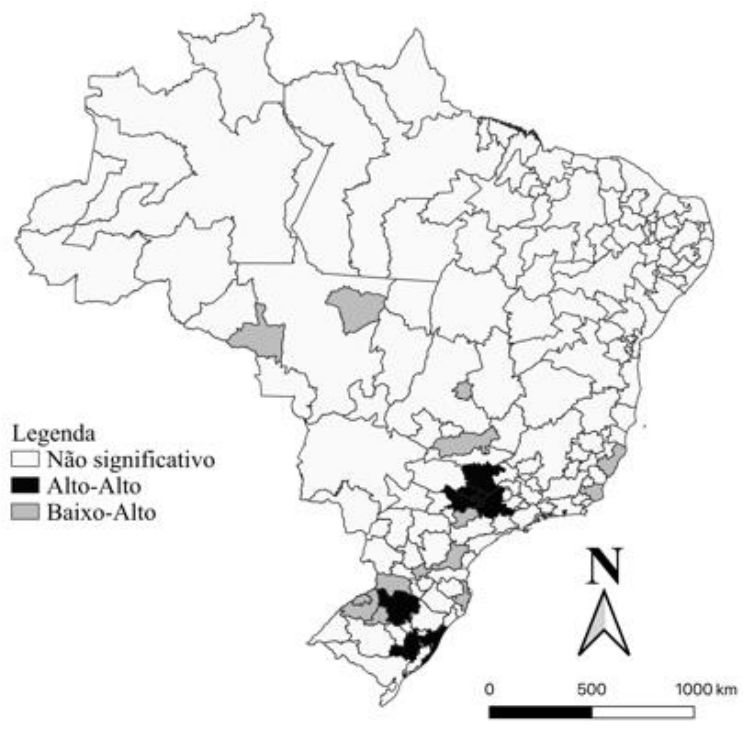

b)

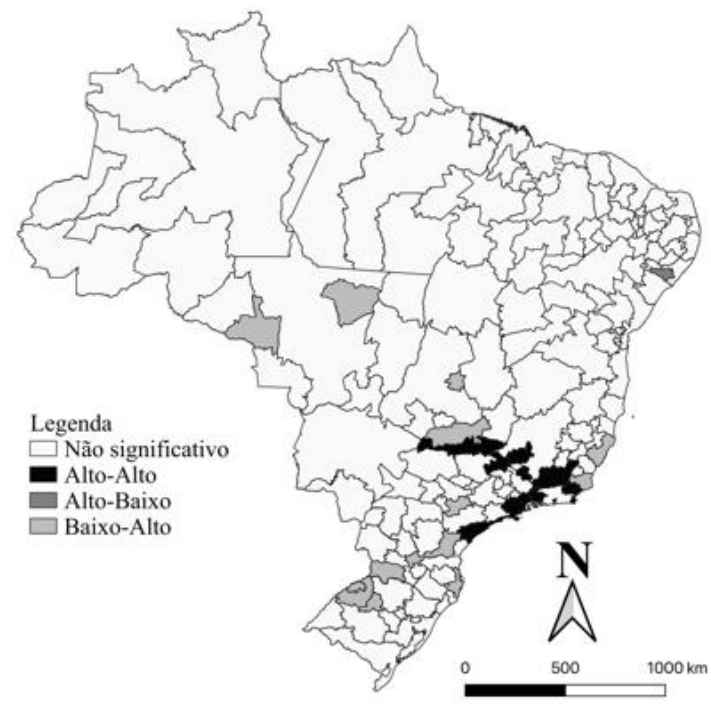

Fonte: Elaborada pelos autores, a partir de dados coletados em Capes (2020), Scopus (2020) e Atlas br (2020).

\section{Discussão}

Barreto et al. (2013) verificou valores médios de índice-h de pesquisadores nas áreas de Saúde Coletiva $(20,4 \pm 10,1)$, Imunologia $(24,0 \pm 6,6)$ e Medicina $(20,5 \pm 6,4)$ no Brasil, próximos aos encontrados para doutorado acadêmico em Biotecnologia. Asnafi et al. (2017) encontraram em equipes editoriais de 62 periódicos internacionais de radiologia um índiceh médio de 26 e participação média de 36 membros, demonstrando que a qualidade de produção e o número de integrantes podem refletir em alto impacto dos periódicos. Doja et al. (2014) verificou em 130 editores de periódicos na área de educação médica que a posição acadêmica influenciou no índice-h, onde a pontuação média de docentes titulares (14) foi maior do que a de docentes associados ou assistentes (7 e 6,5, respectivamente). Tabatabaei-Malazy et al. (2016) classificaram 468 artigos internacionais com a temática de ervas medicinais para diabetes tipo 2 publicados até 2015 e encontraram um índice-h médio de 55. Mester (2016) estudou a faixa dos $25 \%$ pesquisadores mais citados no mundo, com índice-h variando de 169 para Joseph Stiglitz a 251 para Sigmund Freud, observando que muitos daqueles que revolucionaram a ciência ficaram fora desta faixa apesar de alta pontuação geral, caso de Albert Einstein com índice-h de 546. Jacsó (2009) verificou entre 1981 e 2007 que o Brasil apresentou índice-h geral de 214 (Web of Science) ou 216 (Scopus), o mais expressivo da América Latina, com publicações citadas que superaram o somatório total de Argentina, Bolívia, Chile, Colômbia, Equador, Paraguai, Peru, Uruguai e Venezuela. Já um mapa-mundi geral do índice-h construído pelo conjunto de pesquisadores de cada país e citações recebidas entre 1996 e 2010 evidenciou que o Brasil pertenceu a uma faixa de 248 a 336 (Marques, 2013). Relatos de índices-h 
discrepantes podem residir no fato de representarem um grupo muito seleto de pesquisadores, tornando assim os resultados obtidos mais fidedignos por evitar reducionismo da amostra e viés de interpretação.

No Brasil, o uso de indicadores bibliométricos poderia desvelar regionalidades heterogêneas, com alta produtividade acadêmica ou dificuldade de cumprirem as metas nacionais desejáveis aos programas de pós-graduação em Biotecnologia (Brasil, 2017). Nossos achados foram similares a Torres-Freire et al. (2014), que encontraram saturação de pesquisadores em saúde humana na Região Sudeste, e Digiampietri et al. (2019) de pós-doutores em ciências agrícolas, biológicas, da saúde, exatas e da terra e engenharia nas Regiões Sudeste e Sul. Essa concentração é positiva, ao permitir o fortalecimento de centros de referência em pesquisas de fronteira, mas pode diminuir a consolidação de novos centros, a diversificação de saberes e o desenvolvimento local e de longo prazo (Torres-Freire et al., 2014).

A análise espacial ajuda na identificação de determinantes sociais associados a vulnerabilidades em diferentes regionalidades, a exemplo dos estudos que georreferenciaram a densidade populacional de crianças e adolescentes vítimas de violência sexual na Bahia (Nery et al., 2020), portadores de hanseníase de um município hiperendêmico (Macedo et al., 2020a) ou de demais doenças negligenciadas no Piaú (Macedo et al., 2020b). Tal metodologia pode contribuir para vigilância de iniquidades socioeconômicas e problemas de saúde pública no Brasil.

O alto número de titulações em São Paulo equivale a um quarto dos mestres e $40 \%$ dos doutores do país segundo Torres-Freire et al., (2014). Isso pode explicar a formação e concentração de pesquisadores neste Estado junto ao maior IDHM, sugerindo um fenômeno de endogenia acadêmica e consequente previsibilidade de maior índice-h. Ainda, o IDHM de capitais é maior que do que cidades do interior, gerando por parte destas uma dependência tecnológica e de serviços de maior complexidade na área de saúde (Maciel et al., 2020). De forma semelhante, a metade dos programas de pós-graduação deste estudo esteve concentrado em capitais, sugerindo que o grau de desenvolvimento social pode interferir na consolidação de núcleos biotecnológicos no país.

O Brasil ocupa o $62^{\circ}$. lugar no ranking do índice global de inovação 2020, com aumento de difusão e impacto do conhecimento no último ano, apesar de infraestrutura, sofisticação de negócios e trabalhadores do conhecimento serem pilares a desenvolver (Cornell University, Insead e Wipo, 2020). Machado e Bianchetti (2011) trouxeram à luz a perspectiva de relações de poder em educação, ciência e tecnologia, que podem promover o progresso econômico e social em detrimento da Universidade e de seus trabalhadores-pesquisadores, reduzidos a executores e reféns do sistema capitalista. Nesse sentido, a frequência de áreas geográficas com expressão de programas de pós-graduação em Biotecnologia com baixo índice-h e baixo IDHM frente a densidade populacional pode denotar carências de ordem estrutural e processual que merecem ter discussão ampliada por gestores e pela própria comunidade científica, visando construção de malhas colaborativas.

É importante ressaltar que as universidades federais no Brasil nos últimos 10 anos perderam $73 \%$ da verba para construir laboratórios, fazer obras e trocar computadores, o que se torna ainda maior desafiador manter a qualidade do ensino, a alta produtividade e evitar a "fuga de cérebros" de jovens pesquisadores (Tenente, 2020). Em tempos de pandemia por COVID-19, o Brasil foi o terceiro país cujos pesquisadores sentiram maior impacto no trabalho diário, seja por crônico subfinanciamento, atividades remotas obrigatórias ou desacreditação de governantes ou população negacionista, o que aumentou compartilhamento de publicações abertas ou pré-impressões, para a divulgação de evidências ser mais ágil e articulada para diferentes públicos (Castro-Silva e Maciel, 2020; Rijs \& Fenter, 2020).

Lima (2016) verificou que houve o encurtamento dos interstícios médios para titulação em nível de mestrado (de 4 para 2 anos) e doutorado (6,5 para 4 anos) no Brasil, a partir de 1998. Com a atual configuração, o tempo exerce pressão adicional à produtividade que, além do pesquisador, depende das condições de viabilização da pesquisa e da avaliação em pares por periódicos científicos (Rigo, 2017). 
Bendels, Müller, Brueggmann e Groneberg (2018) observaram ainda inequidade de gêneros em periódicos das ciências da vida e da natureza entre 2008 a 2016, onde a autoria feminina ficou subrepresentada em quase 30\% de publicações. Embora não seja objeto do presente estudo, é importante levantar essa questão para minimização de desigualdades latentes na academia.

Como limitações deste estudo, cabe salientar que o impacto científico de indicadores bibliométricos e rankings acadêmicos deve ser visto com parcimônia, para evitar um ambiente de pesquisa disfuncional e nocivo (Adler \& Harzing, 2009). O próprio índice-h é restrito a uma mesma área de conhecimento, pode ser manipulado por meio de autocitações, considerar a literatura cinzenta (como livros) mas não considerar o contexto das citações ou medir a participação individual em autorias coletivas (Marques, 2013). Silva (2019) encoraja uma reflexão multinível, governamental, institucional e individual, para que a produtividade acadêmica não seja apenas performativa, mas valide a melhoria de condições de trabalho e bem-estar do docente e da sociedade.

\section{Conclusão}

O índice-h foi uma ferramenta útil para o mapeamento da produtividade de pesquisadores em Biotecnologia no Brasil. A correlação espacial com desenvolvimento social demonstrou que regiões com maior IDHM convergem com maior índice-h no país. Este estudo contribui para o debate sobre a produtividade acadêmica e a busca de modelos de avaliação dos pesquisadores nesta temática, frente a realidades desiguais no cenário nacional.

Uma sugestão para trabalhos futuros residiria em estudos sobre avaliação da percepção dos atores envolvidos nos Programas stricto sensu em Biotecnologia do país, incluindo docentes, discentes e gestores, visando estimar o impacto locorregional da formação no desempenho de recursos humanos em diferentes realidades socioeconômicas.

\section{Referências}

Adler, N. J. \& Harzing, A. W. (2009). When knowledge wins: Transcending the sense and nonsense of academic rankings. Academy of Management Learning \& Education, 8 (1), 72-95.

Agarwal, A., Durairajanayagam, D., Tatagari, S., Esteves, S. C., Harlev, A., Henkel, R., Roychoudhury, S., Homa, S., Puchalt, N. G., Ramasamy, R., Majzoub, A., Ly, K. D., Tvrda, E., Assidi, M., Kesari, K., Sharma, R., Banihani, S., Ko, E., Abu-Elmagd, M., Gosalvez, J. \& Bashiri, A. (2016). Bibliometrics: tracking research impact by selecting the appropriate metrics. Asian Journal of Andrology, 18 (2), 296-309.

Alcadipani, R. (2017). Periódicos brasileiros em inglês: a mímica do publish or perish “global.” Revista de Administração de Empresas, 57 (4), $405-411$.

Anker, M. S., Hadzibegovic, S., Lena, A. \& Haverkamp, W. (2019). The difference in referencing in Web of Science, Scopus, and Google Scholar. ESC Heart Failure, 6 (6), 1291-1312.

Asnafi, S., Gunderson, T., McDonald, R. J. \& Kallmes, D. F. (2017). Association of h-index of editorial board members and impact factor among radiology journals. Academic Radiology, 24 (2), 119-123.

Atlas br (2020). Atlas do Desenvolvimento Humano no Brasil. Consulta de municípios. http://www.atlasbrasil.org.br/.

Barreto, M. L., Aragão, E., Sousa, L. E. P. F., Santana, T. M. \& Barata, R. B. (2013). Diferenças entre as medidas do índice-h geradas em distintas fontes bibliográficas e engenho de busca. Revista de Saúde Pública, 47 (2), 231-238.

Bendels, M. H. K., Müller, R., Brueggmann, D., Groneberg, D. A. (2018). Gender disparities in high-quality research revealed by Nature index journals. PLoS ONE, 13 (1), e0189136.

Bertoli-Barsotti, L. \& Lando, T. (2017). A theoretical model of the relationship between the h index and other simple citation indicators. Scientometrics, 111 (3), 1415-1448.

Bornmann, L., Marx, W., Gasparyan, A. Y. \& Kitas, G. D. (2012). Diversity, value and limitations of the journal impact factor and alternative metrics. Rheumatology International, 32 (7), 1861-1867.

Brandão, L. C. \& Mello, J. C. C. B. S. (2019). A multi-criteria approach to the h-index. European Journal of Operational Research, 276 (1), $357-363$.

Brasil (2017). Ministério da Educação. Coordenação de Aperfeiçoamento de Pessoal de Nível Superior. Diretoria de avaliação. Documento de área: Biotecnologia. Brasília, DF. 30 p. https://www.gov.br/capes/pt-br/centrais-de-conteudo/copy_of_20122017BIOTECNOLOGIAquadrienal.pdf.

Capes (2020). Coordenação de Aperfeiçoamento de Pessoal de Nível Superior. Plataforma Sucupira. Versão Beta. https://sucupira.capes.gov.br/sucupira/. 
Carpenter, C. R., Cone, D. C. \& Sarli, C. C. (2014). Using publication metrics to highlight academic productivity and research impact. Academic Emergency Medicine, 21 (10), 1160-1172.

Castro-Silva, I. I. \& Maciel, J. A. C. (2020). Panorama de pesquisas com seres humanos sobre COVID-19 no Brasil. Revista Bioética, 28 (4), 655-663.

Ciaccio, E. J., Bhagat, G., Lebwohl, B., Lewis, S. K., Ciacci, C. \& Green, P. H. (2019). Comparison of several author indices for gauging academic productivity. Informatics in Medicine Unlocked, 15, e100166.

Cornell University, Insead \& Wipo (2020). The global innovation index 2020: Who will finance innovation? 13 ed. Ithaca, Fontainebleau and Geneva: Cornell University, Insead \& Wipo. https://www.wipo.int/edocs/pubdocs/en/wipo_pub_gii_2020.pdf.

Cronin, B. \& Meho, L. (2006). Using the h-index to rank influential information scientists. Journal of the American Society for Information Science and Technology, 57 (9), 1275-1278.

De Paula, A. V. \& Boas, A. A. V. (2017). Well-being and quality of working life of university professors in Brazil. In: Boas, A. A. V. Quality of life and quality of working life. Rijeka: InTech.

Digiampietri, L. A., Mugnaini, R., Trucolo, C., Delgado, K. V., Mena-Chalco, J. P. \& Köhler, A. F. (2019). Geographic and disciplinary distribution of the Brazilian's PhD community: patterns of the scientific collaboration structure. Brazilian Journal of Information Science: Research Trends, 13 (4), 113-131.

Dimova, M., Mitnik, A., Suarez-Buitron, P. \& Siqueira, M. (2009). Brazil Biotech Cluster: Minas Gerais. A cluster analysis. Microeconomics of Competitiveness, 1-31. https://www.isc.hbs.edu/Documents/resources/courses/moc-course-at-harvard/pdf/student-projects/Brazil_Biotech_2009.pdf.

Doja, A., Eady, K., Horsley, T., Bould, M. D., Victor, C. J. \& Sampson, M. (2014). The h-index in medical education: an analysis of medical education journal editorial boards. BMC Medical Education, 14, e251.

Hirsch, J. E. (2019). ha: An index to quantify an individual's scientific leadership. Scientometrics, 118, 673-686.

Hirsch, J. E. (2007). Does the h index have predictive power? Proceedings of the National Academy of Sciences of the United States of America, 104 (49), 19193-19198.

Hirsch, J. E. (2005). An index to quantify an individual's scientific research output. Proceedings of the National Academy of Sciences of the United States of America, 102 (46), 16569-16572.

Jacsó, P. (2009). The h-index for countries in Web of Science and Scopus. Online Information Review, 33 (4), 831-837.

León-de la O, D. I., Thorsteinsdóttir, H. \& Calderón-Salinas, J. V. (2018). The rise of health biotechnology research in Latin America: A scientometric analysis of health biotechnology production and impact in Argentina, Brazil, Chile, Colombia, Cuba and Mexico. PLoS ONE, 13 (2), e0191267.

Lima, L. G. A. (2016). A influência da iniciação científica sobre a pós-graduação: um estudo de caso sobre tempo, idade de titulação e produção científica. Dissertação de mestrado, Universidade Federal do Rio Grande do Sul, Porto Alegre, RS, Brasil

Macedo, J. B., Macedo, D. B., Ferreira, A. F., Macedo, G. B., Bortoleto, C. S., Santos, L., Rodrigues, B. V. M. \& Pavinatto, A. (2020a). Hanseníase: determinantes sociais e análise espacial de casos em município hiperendêmico. Research, Society and Development, 9 (10), e5569109010.

Macedo, J. B., Macedo, D. B., Ferreira, A. F., Macedo, G. B., Bortoleto, C. S., Santos, L., Rodrigues, B. V. M. \& Pavinatto, A. (2020b). Análise espacial e determinantes sociais na vigilância das doenças negligenciadas. Research, Society and Development, 9 (8), e808986261.

Machado, A. M. N. \& Bianchetti, L. (2011). (Des)fetichização do produtivismo acadêmico: Desafios para o trabalhador-pesquisador. Revista de Administração de Empresas, 51 (3), 244-254.

Maciel, J. A. C., Castro-Silva, I. I. \& Farias, M. R. (2020). Análise inicial da correlação espacial entre a incidência de COVID-19 e o desenvolvimento humano nos municípios do estado do Ceará no Brasil. Revista Brasileira de Epidemiologia, 23, e200057.

Marques, F. (2013). Os limites do índice-h: supervalorização do indicador que combina qualidade e quantidade da produção científica gera controvérsia. Pesquisa FAPESP, 207, 35-39. https://revistapesquisa.fapesp.br/wp-content/uploads/2013/05/035-039_IndiceH_207.pdf.

Mester, G. (2016). Rankings scientists, journals and countries using h-index. Interdisciplinary Description of Complex Systems, 14 (1), 1-9.

Nery, C. L. P. D., Conceição, M. M., Nery, F. S., Lopes, T. H. C. R., Reis, R. B. \& Felzemburgh, R. D. M. (2020). Caracterização e análise espacial da violência sexual contra crianças e adolescentes na Bahia. Research, Society and Development, 9 (7), e716974661.

Patel, V. M., Ashrafian, H., Almoudaris, A., Makanjuola, J., Bucciarelli-Ducci, C., Darzi, A. \& Athanasiou, T. (2013). Measuring academic performance for healthcare researchers with the H index: which search tool should be used? Medical Principles and Practice, 22 (2), 178-183.

Pereira, A. S., Shitsuka, D. M.; Parreira, F. J. \& Shitsika, R. (2018). Metodologia da pesquisa científica. Santa Maria: UAB/NTE/UFSM. https://repositorio.ufsm.br/bitstream/handle/1/15824/Lic_Computacao_Metodologia-Pesquisa-Cientifica.pdf?sequence=1.

Pnud (2003). Programa das Nações Unidas para o Desenvolvimento. https://www.br.undp.org/content/brazil/pt/home/.

Qgis (2020). QGIS - A Free and Open Source Geographic Information System. https://www.qgis.org/en/site/.

Rigo, A. S. (2017). Comunidade acadêmica, produtivismo e avaliação por pares. Revista de Administração de Empresas, 57 (5), $510-514$.

Rijs, C., Fenter, F. (2020). The academic response to COVID-19: a survey report. Frontiers in Public Health, 8. 
Research, Society and Development, v. 10, n. 1, e29910111807, 2021

(CC BY 4.0) | ISSN 2525-3409 | DOI: http://dx.doi.org/10.33448/rsd-v10i1.11807

Scopus (2020). Search for an author profile. https://www.scopus.com/freelookup/form/author.uri.

Silva, A. B. (2019). Produtivismo acadêmico multinível: mercadoria performativa na pós-graduação em administração. Revista de Administração de Empresas, 59 (5), 341-352.

Simioni, C. L., Dallacorte, C. \& Jacoski, C. A. (2016). Proposição de um índice-h para a produção científica da construção civil nacional. Encontros Bibli: Revista Eletrônica de Biblioteconomia e Ciência da Informação, 21 (46), 137-153.

Tabatabaei-Malazy, O., Ramezani, A., Atlasi, R., Larijani, B. \& Abdollahi, M. (2016). Scientometric study of academic publications on antioxidative herbal medicines in type 2 diabetes mellitus. Journal of Diabetes and Metabolic Disorders, 15 (48), 1-8.

Tenente, L. (2020). G1. Universidades federais perdem, em 10 anos, $73 \%$ da verba para construir laboratórios, fazer obras e trocas computadores https://g1.globo.com/educacao/noticia/2020/08/23/universidades-federais-perdem-em-10-anos-73percent-da-verba-para-construir-laboratorios-fazer-obras-etrocar-computadores.ghtml.

Torres-Freire, C., Golgher, D. \& Callil, V. (2014). Biotecnologia em saúde humana no Brasil: produção científica e pesquisa e desenvolvimento. Novos estudos CEBRAP, 98, 69-93. 